\title{
Organizing Hematoma of the Maxillary Sinus Mimicking Malignancy Diagnosed by Fluorodeoxyglucose Positron-Emission Tomography (FDG PET/CT): A Case Report
}

\author{
Yong Kyun Park ${ }^{1}$; Kyung Soo Kim ${ }^{1, *}$ \\ ${ }^{1}$ Department of Otorhinolaryngology-Head and Neck Surgery, Chung-Ang University Hospital, College of Medicine, Chung-Ang University, Seoul, Korea \\ ${ }^{*}$ Corresponding authors: Kyung Soo Kim, Department of Otorhinolaryngology-Head and Neck Surgery, Chung-Ang University Hospital, College of Medicine, Chung-Ang Univer- \\ sity, P. O. Box: 156-755, Seoul, Korea. Tel: +82-262991765, Fax:+82-28251765, E-mail: 99-21045@hanmail.net
}

Received: March 13, 2014; Revised: June 16, 2014; Accepted: August 2, 2014

\begin{abstract}
Organizing hematoma of the paranasal sinuses is a diagnostic dilemma clinically and radiographically, mimicking benign or malignant neoplastic processes. Although the diagnostic rate of this disease has increased as characteristic imaging findings are somewhat elucidated, endoscopic examination, preoperative biopsy, and computed tomography (CT) imaging do not give helpful information in differentiating these lesions from malignant neoplastic processes.

A 55-year-old man presented with a 4-month history of recurrent nasal bleeding. He also complained of a left-sided nasal obstruction. CT findings were highly suggestive of a malignant tumor of the maxillary sinus. However, based on fluorodeoxyglucose $\mathrm{F}^{18}$ positron-emission tomography (PET/CT) and magnetic resonance imaging (MRI), the provisional diagnosis of benign tumor rather than malignancy was made. Complete resection of the mass was achieved by simple transnasal endoscopic surgery using the Caldwell-Luc approach. Organizing hematoma of the maxillary sinus was diagnosed by histopathologic evaluation. The clinical, radiological, and histopathologic findings of the patient are presented.

In this report, we have presented ${ }^{18}$ FDG-PET findings of organized hematoma of the maxillary sinus (OHMS) that showed an increased FDG uptake in the peripheral rim of the mass with central photopenia. To our knowledge, this is the first case report in the literature reporting FDG-PET/CT findings of OHMS. Careful interpretation of metabolic (FDG-PET/CT) and anatomic (CT and MRI) images should be performed to accurately characterize the expansile lesion of the maxillary sinus in order to increase specificity and reduce equivocal findings significantly.
\end{abstract}

Keywords: Organizing Hematoma; Computed Tomography; Chronic Expanding Hematoma; Fluorodeoxyglucose $\mathrm{F}^{18}$ PositronEmission Tomography; Magnetic Resonance Imaging; Maxillary Sinus

\section{Introduction}

Chronic expanding hematoma (CEH) is a rare presentation of a hematoma characterized by a persistent increase in size for more than a month after the initial hemorrhage (1). CEH presents as a progressively enlarging mass in patients with a history of trauma or surgery because of recurrent intracapsular bleeding from the neovasculature (2). Recently, CEH originating from the paranasal sinuses has been described using two diagnostic terms including "organized hematoma" or "organizing hematoma" which there has been no consensus on diagnostic term of this disease entity (3).

Organizing hematoma of the paranasal sinuses is a diagnostic dilemma clinically and radiographically, mimicking benign, including fungal diseases, or malignant neoplastic processes and causing patients and clinicians undue worry regarding these diagnoses (3). Although the diagnostic rate of this disease entity has been increased, as characteristic imaging findings are somewhat elucidated, endoscopic examination and computed tomography (CT) imaging do not give helpful information in differentiating these lesions from malignant neoplastic processes (3). Also, because distinct pathological differences are observed between the basal and peripheral portions of organizing hema-

Copyright ( 2015 , Tehran University of Medical Sciences and Iranian Society of Radiology. This is an open-access article distributed under the terms of the Creative Commons Attribution-NonCommercial 4.0 International License (http://creativecommons.org/licenses/by-nc/4.0/) which permits copy and redistribute the material just in noncommercial usages, provided the original work is properly cited. 
tomas, preoperative biopsy taken from the periphery may usually be inconclusive (4). Recently, magnetic resonance imaging (MRI) can lead to positive diagnosis by recognizing the distinct outer rims, called "shells" of T2 hypointensity typically seen in these lesions (3). However, the most important limitation of MRI is that patients with severe claustrophobia may not be able to tolerate MRI procedures in closed gantry systems.

Positron-emission tomography (PET) with F18-fluorodeoxyglucose (FDG) is an evolving diagnostic modality used for tumor detection, staging, therapeutic monitoring, and follow-up of various malignant tumors. Only five reports about ${ }^{18}$ FDG-PET imaging features of CEHs originating from other sites of the body have been documented (5-9). To the best of our knowledge, ${ }^{18} \mathrm{FDG}$-PET images of organizing hematoma of the maxillary sinus have not been previously reported. In this report, we present ${ }^{18} \mathrm{~F}$-FDG-PET/CT findings in organizing hematoma of the maxillary sinus (OHMS) compared to concurrent $\mathrm{CT}$ and MRI findings.

\section{Case Presentation}

A 55-year-old man presented with a 4-month history of recurrent nasal bleeding. He also complained of a leftsided nasal obstruction. He had no history of antecedent trauma, nasal surgery, or bleeding diatheses and no facial numbness or pressure. His medical and family histories were noncontributory. On physical examination, no facial deformity and eye movement limitation was detected. His complete hematology profile and coagulation tests were normal. On endoscopic examination, the left lateral nasal wall was medially displaced towards the nasal septum, blocking the nasal passage completely. So, it was not possible to examine the nasal cavity endoscopically because of the narrowed passage. After shrinkage of the inferior turbinate mucosa, we observed a lobulated pinkish mass in the inferior meatus that caused medial displacement of the inferior turbinate (Figure 1). Preoperative biopsy was performed from the mass of the inferior meatus, but the results demonstrated only vascular fibrous tissue with chronic inflammation. Enhanced CT of the paranasal sinuses showed a $4.3 \times 2.8 \times 4.3 \mathrm{~cm}$ sized heterogeneous enhanced soft tissue mass in the maxillary sinus, the central region of which was in particular strongly enhanced (Figure 2). The expansion of the maxillary sinus and bony thinning of the posterior and medial wall of the left maxillary sinus were also noted. The mass caused obstruction of the left nasal cavity with medial displacement of the middle and inferior turbinate. CT findings were highly suspicious for a malignant tumor of the maxillary sinus. He simultaneously underwent MRI and whole-body combined ${ }^{18} \mathrm{~F}-\mathrm{PET} / \mathrm{CT}$ for evaluation of a malignant tumor of the maxillary sinus. T1- weighted MRI images revealed a large, lobulated mass that was mostly isodense to the inferior turbinate diffusely interspersed with hyperintensity. T2-weighted images showed the marked heterogeneity of the lesion with a mix of hypointense (peripheral part), isointense, and hyperintense (central part) signals and a dark peripheral rim surrounding the lesion. Bright signal intensity due to mucosal inflammation of the involved maxillary sinus was distinguished from the lesion on T2-weighted images (Figure 3).

Integrated ${ }^{18} \mathrm{~F}$-FDG-PET/CT images showed moderate uptake only at the peripheral rim of the mass with negative FDG uptake in the central portion. The maximum standardized uptake value (SUV) of this lesion was 3.80. Other signs of abnormal uptake suggesting a malignant lesion were not observed (Figure 4).

Based on PET/CT and MRI, the provisional diagnosis of benign tumor rather than malignancy was made. Complete resection of the mass was achieved by simple transnasal endoscopic surgery with Caldwell-Luc approach. Histologically, fibrous tissue, neovascularization, and extravasated red blood cells in the subepithelium with no evidence of malignancy is a combination characteristic of organizing hematoma. The patient's postoperative course was uneventful and his symptoms improved markedly. There has been no sign of recurrence 1 year after the operation.

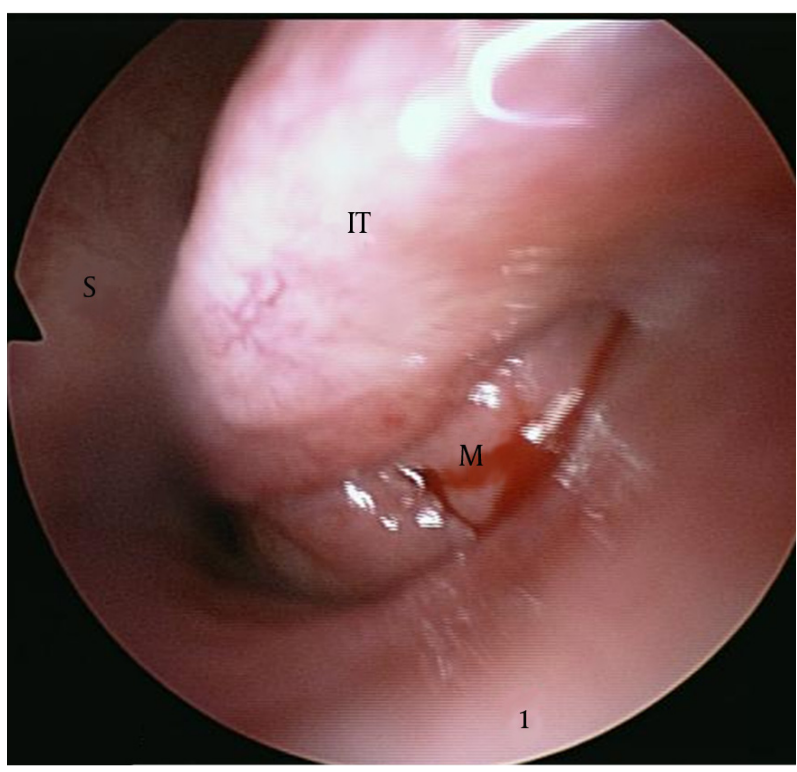

Figure 1. A 55-year-old man with a history of recurrent nasal bleeding and obstruction. After shrinkage of the inferior turbinate mucosa, we observed a lobulated pinkish mass in the inferior meatus that caused medial displacement of the inferior turbinate. (M: mass, S: septum, IT: inferior turbinate) 

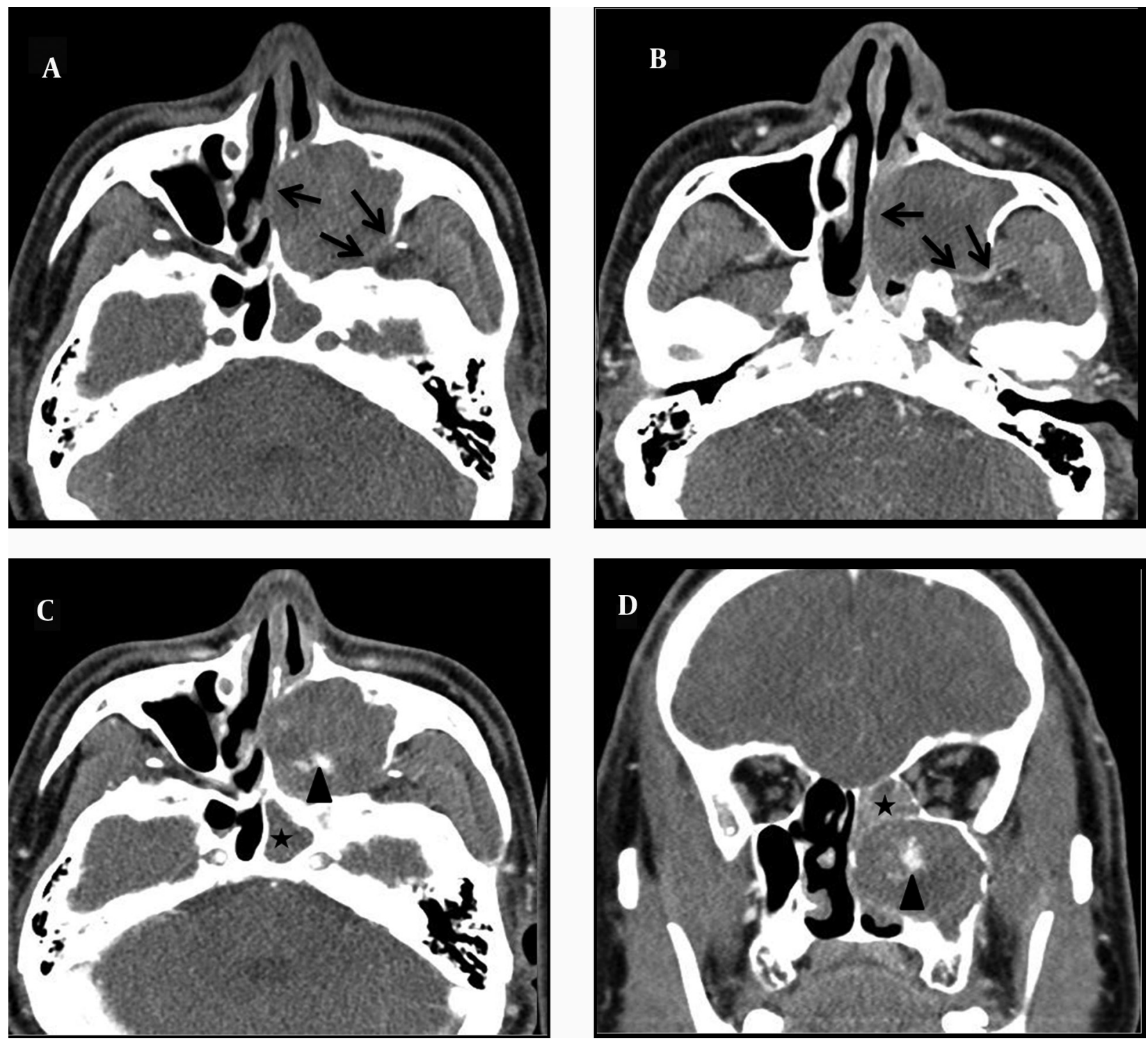

Figure 2. Enhanced CT of the paranasal sinuses shows a $4.3 \times 2.8 \times 4.3$ sized heterogeneous enhanced soft tissue mass in the maxillary sinus, the central region of which was in particular strongly enhanced (arrow head). The expansion of the maxillary sinus and bony thinning of the posterior and medial wall of the left maxillary sinus are also noted (black arrows). A, nonenhanced axial section. B and C, enhanced axial sections. D, enhanced coronal section. Also, CT scans showed the opacification of the sphenoid and ethmoid sinuses because of the mass obstructing the draining sinus ostia (asterisk).

\section{Discussion}

$\mathrm{CEH}$ can occur in a variety of locations. Although most hematomas resolve spontaneously, a few can persist for long periods forming slowly expanding space-occupying masses (1). This expanding process is attributable to the irritant effects of blood and the products of its breakdown, which induce repeated episodes of bleeding from the capillaries in the granulation tissue (10). Sinonasal organizing (organized) hematoma is a rare type of $\mathrm{CEH}$ originating from the paranasal sinuses whose clinical characteristics lead to be misdiagnosed as a malignant or locally aggressive neoplasm (3). The number of reported cases has dramatically risen since 2005 because of elucidation of the characteristic imaging findings and the specific pathologic findings (11-15). Because the maxillary sinus is the largest paranasal sinus that allows negative pressure, significant leakage of blood may develop in the nasal cavity into the sinus through the ostium (15). Therefore, organizing hematoma most commonly affects the maxillary sinus and is known as OHMS (11). 
Park YKet al.
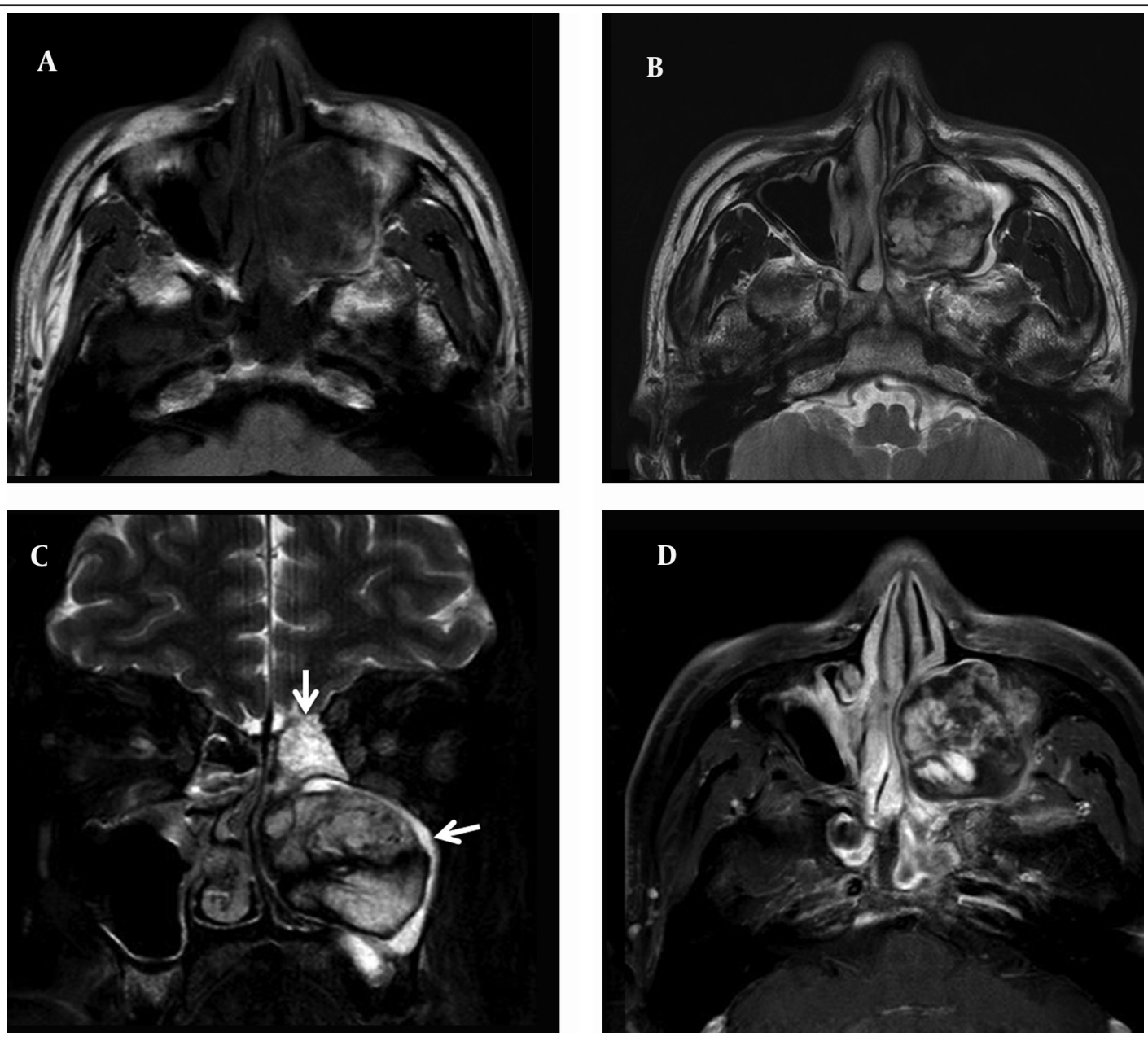

Figure 3. A, Axial T1-weighted T1-weighted MR image shows that the lesion is mostly isodense to the inferior turbinate interspersed with hyperintesity. B, Axial T2-weighted MR image shows marked heterogeneity of the lesion with a mix of hypointense (peripheral part), isointense, and hyperintense (central part) signals and a dark peripheral rim surrounding the lesion. C, Coronal, fat-suppressed, T2-weighted MR image shows the marked heterogeneity of the lesion with a mix of hypointense, isointense and hyperintense signals. A dark peripheral rim surrounding the lesion and a bright signal intensity due to mucosal inflammation of the involved maxillary sinus and ethmoid sinus (white arrows) is well demonstrated. D, Contrast-enhanced axial, fatsuppressed, T1-weighted MR image shows a marked papillary enhancement within the lesion.
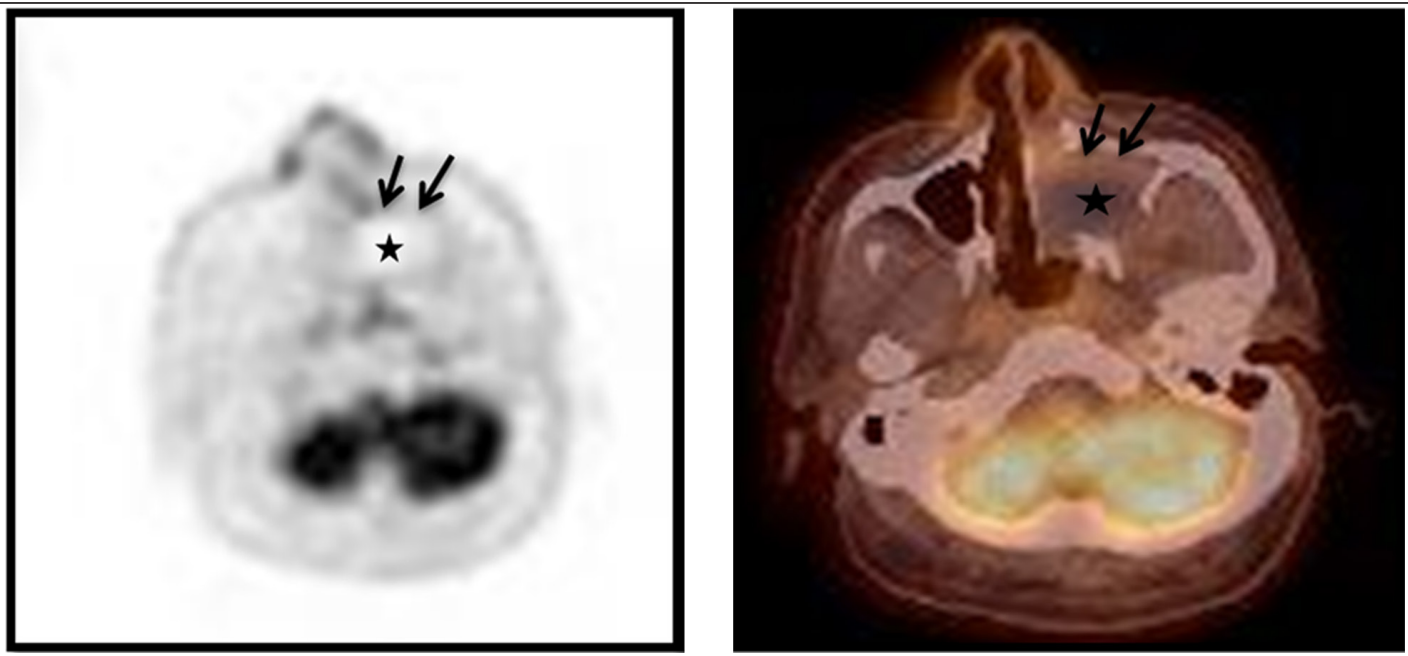

Figure 4. Integrated ${ }^{18}$ F-FDG-PET/CT images show moderate uptake only at the peripheral rim (black arrows) of the mass with negative FDG uptake in the central portion (asterisk). The maximum standardized uptake value (SUVmax) of this lesion is 3.80. Other signs of abnormal uptake suggesting a malignant lesion are not observed. 
Although diagnostic criteria for correct identification of OHMS are not well known (3), corrective preoperative diagnosis is important to avoid unnecessary extensive surgery because this condition is curative with a simple, conservative endoscopic approach and rarely recurs (11). The reported CT findings of organizing hematoma include an expansive, clearly demarcated and compressive mass causing bony erosion without a destructive feature (14). After administration of contrast, heterogeneous enhancement in a patchy distribution is usually found within the lesion $(15,16)$. MRI is superior to CT for determining the margin and extent of the lesion. In MR images, it has biphasic appearance in which the central part of the lesion presents as low intensity on T1-weighted images and high intensity on T2-weighted images, corresponding with areas of fresh hemorrhage and the peripheral part is less enhanced with a zone of fibrosis, especially called as "shells" of T2 hypointensity which is the most diagnostic finding on MRI (3, $14,17)$. However, if it is not possible to differentiate benign tumors from malignancy by CT and MRI, or if the patient cannot perform MRI scan because of severe claustrophobia, definite preoperative diagnosis is difficult.

CEHs can be misdiagnosed as malignant tumors because of their large size and slow, progressive enlargement $(5,7)$. Although ${ }^{18}$ FDG-PET images of CEH are not widely available, ${ }^{18}$ FDG-PET shows moderate FDG uptake, the maximum standardized uptake value (SUVmax) of 3.1 to 5.5 , only at the periphery of the mass with central photon defects that is probably characteristic of $\mathrm{CEH}$, suggesting chronic inflammation or granulation tissue in the fibrous wall (5-9). If the SUVmax were set to a cutoff point of 3.0 to distinguish between benign and malignant tumors, these lesions could be misdiagnosed as malignant tumors (5). However, the use of FDG-PET imaging for tumor diagnosis is limited by the fact that FDG, a glucose analog, is taken up not only by tumor cells but also by macrophages and immature granulation tissue containing fibroblasts and inflammation (18). CEH has abundant granulation tissue with neovascularization, macrophages and inflammation, which corresponds to organizing processes. These pathological changes are considered to have resulted in increased FDG uptake within the organizing hematoma (5-9).

In our patient, ${ }^{18}$ FDG-PET images of the lesion revealed central photon defects with moderately increased FDG uptake in the peripheral rim of the space-occupying mass in the left maxillary sinus. The SUVmax of the lesion was 3.8. This is consistent with previous reports of ${ }^{18}$ FDGPET imaging of CEH. Although the FDG uptake pattern might be seen if a malignant tumor has a tendency of central necrosis, the characteristics of FDG-PET images of OHMS and CEH, including FDG uptake in the peripheral rim with central photon defect of the mass as a result of inflammation should be recognized as a potential interpretive pitfall that mimics a malignant tumor (5).

In summary, we have presented ${ }^{18}$ FDG-PET findings of OHMS that showed an increased FDG uptake in the pe- ripheral rim of the mass with central photopenia. To our knowledge, this is the first report in literature reporting ${ }^{18}$ FDG-PET findings of OHMS with SUVmax values suggestive of a malignancy. Careful interpretation of metabolic (FDG-PET/CT) and anatomic (CT and MRI) images should be performed to accurately characterize the expansile lesion of the maxillary sinus in order to increase specificity and reduce equivocal findings significantly.

\section{Authors' Contributions}

Yong Kyun Park was responsible for drafting, editing, and literature search. Kyung Soo Kim was responsible for paper design, editing, and final approval.

\section{References}

1. Reid JD, Kommareddi S, Lankerani M, Park MC. Chronic expanding hematomas. A clinicopathologic entity. JAMA. 1980;244(21):2441-2.

2. Ooba H, Kamida T, Isono M, Kobayashi H, Karashima A, Yamashita M. Chronic intraventricular encapsulated hematoma presenting unique radiological features: a case report. Surg Neurol. 2003;59(1):23-7.

3. Wu AW, Ting JY, Borgie RC, Busaba NY, Sadow PM, Juliano AF, et al. Diagnostic characteristics of sinonasal organizing hematomas: avoiding misdiagnosis. Int Forum Allergy Rhinol. 2013;3(7):598-602.

4. Urata S, Ohki M, Tsutsumi T, Kikuchi S. Organised haematoma of the maxillary sinus: pathophysiological differences suggesting a new aetiological hypothesis. J Laryngol Otol. 2013;127(5):519-24.

5. Hamada K, Myoui A, Ueda T, Higuchi I, Inoue A, Tamai N, et al. FDG PET imaging for chronic expanding hematoma in pelvis with massive bone destruction. Skeletal Radiol. 2005;34(12):807-11.

6. Kwon YS, Koh WJ, Kim TS, Lee KS, Kim BT, Shim YM. Chronic expanding hematoma of the thorax. Yonsei Med J.2007;48(2):337-40.

7. Takahama M, Yamamoto R, Nakajima R, Izumi N, Tada H. Extrathoracic protrusion of a chronic expanding hematoma in the chest mimicking a soft tissue tumor. Gen Thorac Cardiovasc Surg. 2010;58(4):202-4.

8. Tokue H, Tokue A, Okauchi K, Tsushima Y. 2-[(1)(8)F]fluoro2-deoxy-D-glucose (FDG) positron-emission tomography (PET) findings of chronic expanding intrapericardial hematoma: a potential interpretive pitfall that mimics a malignant tumor. $J$ Cardiothorac Surg. 2013;8:13.

9. Hata Y, Sakamoto S, Shiraga N, Sato K, Sato F, Otsuka H, et al. A case of chronic expanding hematoma resulting in fatal hemoptysis. JThorac Dis. 2012;4(5):508-11.

10. Labadie EL, Glover D. Physiopathogenesis of subdural hematomas. Part 1: Histological and biochemical comparisons of subcutaneous hematoma in rats with subdural hematoma in man. $J$ Neurosurg. 1976;45(4):382-92.

11. Song HM, Jang YJ, Chung YS, Lee BJ. Organizing hematoma of the maxillary sinus. Otolaryngol Head Neck Surg. 2007;136(4):616-20.

12. Nishiguchi T, Nakamura A, Mochizuki K, Tokuhara Y, Yamane $H$, Inoue Y. Expansile organized maxillary sinus hematoma: MR and CT findings and review of literature. AJNR Am J Neuroradiol. 2007;28(7):1375-7.

13. Yagisawa M, Ishitoya J, Tsukuda M. Hematoma-like mass of the maxillary sinus. Acta Otolaryngol. 2006;126(3):277-81.

14. Omura G, Watanabe K, Fujishiro Y, Ebihara Y, Nakao K, Asakage T. Organized hematoma in the paranasal sinus and nasal cavity-imaging diagnosis and pathological findings. Auris Nasus Larynx. 2010;37(2):173-7.

15. Lee HK, Smoker WR, Lee BJ, Kim SJ, Cho KJ. Organized hematoma of the maxillary sinus: CT findings. AJR Am J Roentgenol. 2007;188(4):W370-3.

16. Tabaee A, Kacker A. Hematoma of the maxillary sinus presenting as a mass-a case report and review of literature. Int J PediatrOtorhinolaryngol. 2002;65(2):153-7. 


\section{Park YKet al.}

17. Kim EY, Kim HJ, Chung SK, Dhong HJ, Kim HY, Yim YJ, et al. Sinonasal organized hematoma: CT and MR imaging findings. AJNR Am J Neuroradiol. 2008;29(6):1204-8.
18. Schulte M, Brecht-Krauss D, Heymer B, Guhlmann A, Hartwig E, Sarkar MR, et al. Grading of tumors and tumorlike lesions of bone: evaluation by FDG PET. J Nucl Med. 2000;41(10):1695-701. 